\title{
Eosinophilic Fascitis: A Rare Fibrosing Disorder
}

\author{
Paudyal BP, ${ }^{1}$ Gyawalee $\mathrm{M}^{2}$ Sigdel $\mathrm{K}^{1}$
}

\author{
${ }^{1}$ Department of Internal Medicine \\ ${ }^{2}$ Department of Dermatology \\ Patan Hospital, Patan Academy of Health Sciences \\ Kathmandu, Nepal
}

Corresponding Author

Buddhi Paudyal

Department of Internal Medicine

Patan Academy of Health Sciences

Patan Hospital, Lalitpur, Nepal

Email: buddhipaudyal@yahoo.com

\begin{abstract}
Eosinophilic fasciitis is a rare systemic disease characterized by inflammatory thickening of the skin and underlying fascia, peripheral eosinophilia, elevated inflammatory markers and typical biopsy findings. Internal organ involvement is rare unlike in other fibrosing disorders. Though this is usually a benign disease, it is often difficult to diagnose and the course may also be variable. Glucocorticoids are the mainstay of therapy, but in many cases steroid sparing immunosuppressive drugs are required particularly if the diseases has protracted course. The author reports a typical case of this rare disorder which could well be a first report from Nepal.
\end{abstract}

\section{KEYWORDS}

Eosinophilic Fascitis, fibrosing disorders

Citation

Paudyal BP, Gyawalee M, Sigdel K. Eosinophilic Fascitis: A Rare Fibrosing Disorder. Kathmandu Univ Med J 2012;39(3):73-75.

\section{INTRODUCTION}

Eosinophilic fasciitis (EF) is an uncommon disorder with unknown etiology and a poorly understood pathogenesis. It is an unusual fibrosing disorder of skin and underlying fascia of the trunk and extremities closely mimicking some of the clinical features of systemic sclerosis. It was first described by Shulman in 1974 as 'diffuse fascitis'; one year later Rodnan introduced the current term for this syndrome. EF typically presents with painful swellings over the limbs, thickening of skin and underlying tissues, arthritis, and peripheral blood eosinophilia, but with relative sparing of the visceral structures. ${ }^{1}$ We present a case of this rare rheumatic syndrome with typical clinical manifestations with blood eosinophilia and positive biopsy findings.

\section{CASE REPORT}

A 28 year old school teacher presented to us with six months' history of painful extremities that started from left knee and gradually spread to involve other areas particularly right knee, both forearms and shoulders in a few months' time. The pain was accompanied by oedema and tightness of muscles of the thighs, calves, forearms and arms, and anterior abdominal wall. The tightness of muscles of the lower extremities led him difficulty in walking and unable to sit in the squatting position. There was no associated fever, muscle weakness, Raynaud's phenomenon, oral ulcers, skin rashes, hair loss, shortness of breath, haemoptysis, and bowel and bladder abnormalities. Examination revealed tight skin and underlying tissues in abdominal wall, forearms, thighs, and calves with reddening of the overlying skin in some of these areas. The left knee and both ankles were inflamed. There were multiple hypopigmented skin lesions in the left thigh. The left knee was in a state of partial flexion due to tightness of the skin and underlying tissues (fig 1).

There was no sclerodactyly; and the muscle power was normal in all extremities. Systemic examination was remarkably normal. Investigations revealed normal white blood count $(7600 / \mu \mathrm{L})$ with high eosinophils $(10 \%)$, and normal ESR. The rest of routine tests including $\mathrm{Hb}$, platelets, 


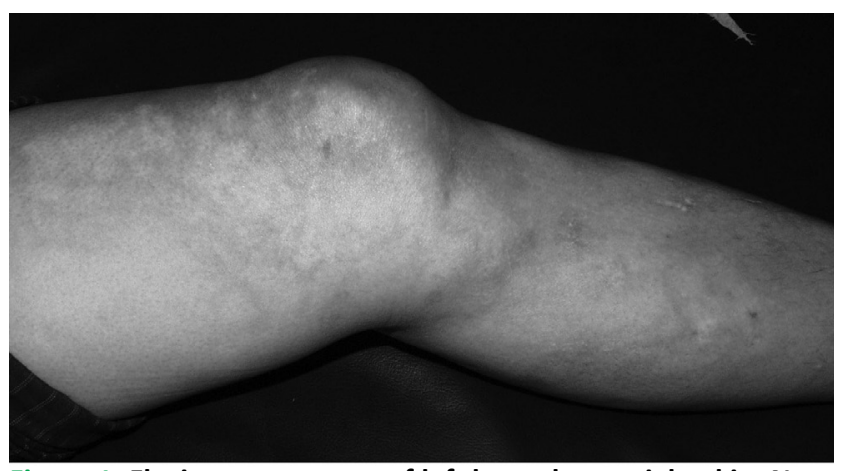

Figure 1. Flexion contracture of left knee due to tight skin. Note hypopigmented skin lesions in and around the knee.

random blood sugar, electrolytes, aspartate transaminase, creatinine, creatinine kinase, thyroid function tests, ECG, and chest $\mathrm{x}$-ray were normal. Full thickness biopsy from the right thigh showed periadnexal inflammation in the subepithelium, and lymphoplasmacytic infiltrations in the fibrous septa surrounding the adipose tissue and in skeletal muscles (fig 2).

Based on the clinical features, peripheral eosinophilia, and biopsy findings, a diagnosis of EF was established and the patient was started on tapering dose of Prednisolone (starting $40 \mathrm{mg} /$ day) and Methotrexate as a steroid sparing agent. When seen at six months for follow up, the skin tightness and pain were much improved and he was walking normally. Moreover there was no peripheral eosinophilia.

\section{DISCUSSION}

EF is an infrequent disease; until now only a few hundred cases have been reported in the medical literature so far. It is characterized by acute or subacute symmetric swelling of the skin and the subcutaneous tissues of the forearms, flanks, and upper part of legs; while the hands, face, and viscera are usually spared. ${ }^{2}$

The etiology of the EF is not clearly known, though a few associations have been suggested. In almost $50 \%$ of cases, clinical manifestations of EF may be preceded by strenuous physical exercise and trauma. ${ }^{3}$ Other causal associations include initiation of haemodialysis, infection with Borrelia borgdorferi, and exposure to L- tryptophan which used to be employed as a dietary supplement and antidepressant in the past. Historically, L-tryptophan ingestion had been related with Eosinophilia-myalgia syndrome, a distinct clinical condition that mimics many clinical features of EF. Though we could not test for Borrelia serology because of unavailability, none of the other risk factors were present in our patient.

EF seems to be equally common in both sexes, though some reports say it is twice more common in males; it is more often seen in $4^{\text {th }}$ to $7^{\text {th }}$ decade with the mean age of onset 47 years. ${ }^{4,5}$

The pathogenesis and natural history of EF remains unclear as clinical spectrum is still evolving with addition of new

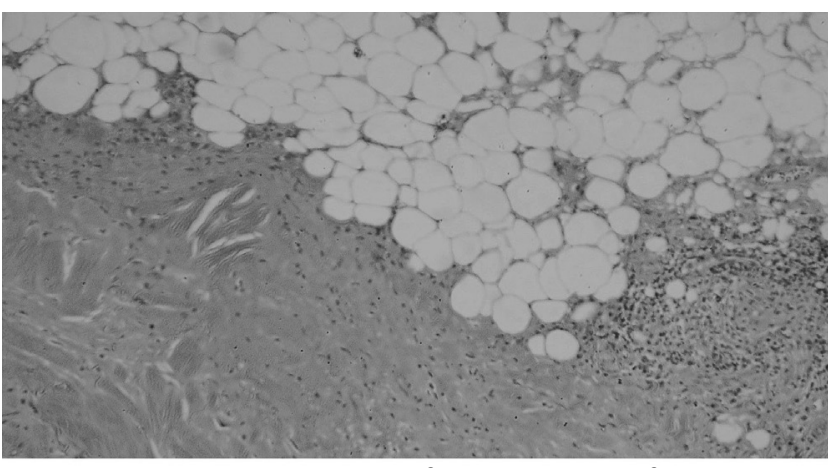

Figure 2.Lymphoplasmacytic infiltrates in the fibrous septa surrounding the adipose tissue and in skeletal muscle.

findings in the literature. Tissue and peripheral blood eosinophilia has been suggested to have a causal role in fibrosis through generation of various inflammatory proteins viz. transforming growth factor $\beta$ (TGF $\beta$ ), eosinophilic cationic protein, and interleukin-5. ${ }^{1,5}$ The disease course can be variable: many patients achieve remission either spontaneously or with steroids, while others experience a recurrent disease with frequent relapses, and few others develop a long term illness. ${ }^{6}$ When seen at six months, our patient was much better with decrease in the pain and swelling in the extremities, absence of joint inflammation, and normalization of peripheral blood eosinophilia.

A diagnostic criteria for EF has been suggested; and this includes following three characteristic features. ${ }^{1}$

- $\quad$ Soft tissue sclerosis and fibrosis, initially developing in extremities, sparing hands and feet

- $\quad$ Absence of Raynaud's sign and visceral involvement (unlike in scleroderma)

- $\quad$ Lymphoplasmatic infiltration of the fascia with a variable number of eosinophils without necrotic vascular lesions.

This criteria primarily helps to differentiate EF from scleroderma, as many researchers still believe that the former is a variant of the later. However, there are significant differences that seem to set EF apart from systemic sclerosis. These include the relative absence of Raynaud's phenomenon, normal nailfold capillaries, infrequent visceral involvement, and absence of the serological features which characterize systemic sclerosis. ${ }^{7}$ The diagnostic dilemma can be solved by an elliptical full thickness skin to muscle biopsy that shows typical findings in patients with EF. Tissue eosinophilia, however, may not be present in many patients with $\mathrm{EF}$, as exemplified by our own patient. MRI, on the other hand, can be very useful to confirm the diagnosis, to monitor the response to therapy and to evaluate the relapse of the disease. ${ }^{5}$

Musculoskeletal manifestations of EF include induration of skin and subcuataneous fat with subsequent peau d' orange appearance, hypo- or hyperpigmentation of the skin and peripheral joint arthritis. Approximately $25 \%$ of the patients develop carpal tunnel syndrome during the course of the disease. ${ }^{2}$ Though less frequent, visceral involvement can still 
occur in EF, and include conditions like splenomegaly, lung restriction, esophageal dysmotility, peripheral neuropathy, autoimmune thyroiditis, and haematological diseases like aplastic anemia, hemolytic anemia, thrombocytopenia, and various lympho- and myeloproliforetive diseases. ${ }^{2,1}$

Common laboratory abnormalities in EF include peripheral blood eosinophilia, polyclonal hypergammaglobulinemia, increased inflammatory markers (i.e., erythrocyte sedimentation rateandC-reactive protein), and, occasionally, elevated muscle enzymes (creatine phosphokinase and aldolase) suggesting the presence of underlying muscular involvement. Peripheral blood eosinophilia is present in up to $80 \%$ of cases; however this is not a prerequisite for diagnosis. ${ }^{5}$ Serological tests like antinuclear antibodies are rarely positive, and scleroderma- specific autoantibodies are usually absent. ${ }^{7}$

Corticosteroids are the first-line treatment for EF and usually effective in more then $70 \%$ of patients. Steroid sparing drugs like hydroxychloroquine, methotrexate, and d-penicillamine are often employed to prevent steroid side effects as treatment course is often protracted. ${ }^{5}$

\section{REFERENCES}

1. Bobrowska-Snarska D, Ostanek L, Brzosko M. Fasciitis eosinophilica: personal observations and a review of the literature. Pol Arch Med Wewn 2007;117:184-191.

2. Danis $R$, Akbulut $S$, Altintas A, Ozmen1 S,Ozmen CA. Unusual presentation of eosinophilic fasciitis: two case reports and a review of the literature. Journal of Medical Case Reports 2010,4:46.

3. Killen JWW, Swift GL,White RJ. Eosinophilic fasciitis with pulmonary and pleural Involvement. Postgrad Med J 2000;76:36-37.

4. Lakhanpal S, Ginsburg WW, Michet CJ, Doyle JA, Moore SB. Eosinophilic fasciitis: clinical spectrum and therapeutic response in 52 cases. Semin Arthritis Rheum 1988;17:221-31.

5. Boin F, Hummers LK. Scleroderma-like Fibrosing Disorders. Rheum Dis Clin N Am 2008;34:199-220.

6. Seibold JR. Scleroderma in Kelly's Textbook of Rheumatology. Harris ED, Budd RC, Genovese MC, Firenstein GC, Sargent JS, Sledge CB, eds. 7th edition. 2005. pps 1302-1303.

7. Maddison PJ. Eosinophilic Fascitis. British Journal of Rheumatology 1990;29:81-83. 